\title{
High on-treatment platelet reactivity on commonly prescribed antiplatelet agents following transient ischaemic attack or ischaemic stroke: results from the Trinity Antiplatelet Responsiveness (TRAP) study
}

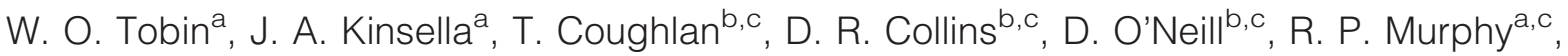 \\ B. Egan ${ }^{d}$, S. Tierney ${ }^{d}$, T. M. Feeley ${ }^{d}$ and D. J. H. McCabe Me,ce $^{\text {a,c }}$ \\ ${ }^{a}$ Department of Neurology, Adelaide and Meath Hospital, Dublin, incorporating the National Children's Hospital, Trinity College Dublin; \\ ${ }^{\mathrm{b}}$ Department of Age Related Health Care, Adelaide and Meath Hospital, Dublin, incorporating the National Children's Hospital, Trinity \\ College Dublin; ${ }^{\mathrm{c}}$ Stroke Service, Adelaide and Meath Hospital, Dublin, incorporating the National Children's Hospital, Trinity College \\ Dublin; ${ }^{\mathrm{d} D e p a r t m e n t ~ o f ~ V a s c u l a r ~ S u r g e r y, ~ A d e l a i d e ~ a n d ~ M e a t h ~ H o s p i t a l, ~ D u b l i n, ~ i n c o r p o r a t i n g ~ t h e ~ N a t i o n a l ~ C h i l d r e n ' s ~ H o s p i t a l, ~ T r i n i t y ~}$ \\ College Dublin, Dublin, Ireland; and ${ }^{\mathrm{e} D e p a r t m e n t ~ o f ~ C l i n i c a l ~ N e u r o s c i e n c e s, ~ R o y a l ~ F r e e ~ C a m p u s, ~ U C L ~ I n s t i t u t e ~ o f ~ N e u r o l o g y, ~ L o n d o n, ~}$ \\ $U K$
}

\section{Keywords:}

aspirin, clopidogrel, flow cytometry, high on-treatment platelet reactivity, platelet function analyser-100, stroke, transient ischaemic attack

Received 29 April 2012 Accepted 25 July 2012
Background and purpose: The prevalence of ex vivo 'high on-treatment platelet reactivity' (HTPR) to antiplatelet regimens in patients with ischaemic cerebrovascular disease (CVD) is uncertain.

Methods: HTPR was assessed with PFA-100 collagen-epinephrine (C-EPI) and collagen-ADP (C-ADP) cartridges. Platelet activation (CD62P, CD63 and leucocyte -platelet complex formation) was assessed with whole-blood flow cytometry. Patients were assessed at baseline $[\leq 4$ weeks of transient ischaemic attack (TIA) or ischaemic stroke], and at 14 days and $\geq 90$ days after changing treatment from (i) no medication to aspirin monotherapy $(N=26)$ or (ii) aspirin to clopidogrel monotherapy $(N=22)$. HTPR was defined in a novel, 'longitudinal fashion' as failure to prolong relevant closure times compared with the patient's 'baseline value' before he/she underwent an antiplatelet change by more than twice the coefficient of variation of the assay.

Results: (i) C-EPI closure times increased at 14 days and 90 days after commencing aspirin $(P=0.002) ; 24 \%$ at 14 days and $18 \%$ at 90 days demonstrated HTPR on aspirin. (ii) C-ADP closure times increased at 14 days $(P=0.001)$ but not 90 days $(P=0.09)$ after changing from aspirin to clopidogrel; $41 \%$ at 14 days, and $35 \%$ at 90 days demonstrated HTPR on clopidogrel. Platelet activation was unaffected by aspirin $(P=0.09)$. The percentage neutrophil-platelet complexes decreased at 14 days $(P=0.02)$, but this reduction was not maintained 90 days after changing to clopidogrel $(P=0.3)$. No patient had a recurrent vascular event during prospective follow-up.

Conclusions: Longitudinal definitions of HTPR in patients with ischaemic CVD who are undergoing a change in antiplatelet therapy have the potential to provide more clinically meaningful information than traditional 'cross-sectional definitions' of HTPR which are usually based on the comparison of patients' values with those in healthy controls. Using our novel, longitudinal definition of HTPR, the PFA-100 could be used to monitor ex vivo responsiveness to aspirin, and larger, prospective studies are warranted to assess the clinical predictive value of this and other platelet function tests in patients with ischaemic CVD.
Correspondence: D. McCabe, Department of Neurology, The Adelaide and Meath Hospital, Dublin, incorporating the National Children's Hospital, Dublin 24, Ireland (tel.: +353 14144217 ; fax: +353 14143031 ; e-mail:dominick.mccabe@amnch.ie).

\section{Introduction}

Antiplatelet agents play a key role in secondary prevention in ischaemic cerebrovascular disease (CVD), but there are no routinely used laboratory tests to 
guide optimal antiplatelet treatment in individuals, akin to the INR in patients on warfarin. Data on the prevalence of ex vivo 'high on-treatment platelet reactivity' (HTPR) at high shear stress on the platelet function analyser (PFA-100 ${ }^{\circledR}$; Dade-Behring, Milton Keynes, UK) are limited in ischaemic CVD. Most studies define patients as having HTPR based on 'cross-sectional, case-control' definitions whereby patient results are compared with those from a group of controls [1-10]. Aspirin has been shown to prolong collagen-epinephrine closure times in patients with transient ischaemic attack (TIA) or ischaemic stroke, alone $[1,11,12]$ and in combination with clopidogrel $[10,11]$. There are conflicting data regarding the ability of the PFA-100 to reliably detect HTPR on long-term clopidogrel $[9,10]$. Few well-designed longitudinal studies, which have the advantage of patients acting as their own baseline controls [6], have assessed the impact of starting aspirin, or changing from aspirin to clopidogrel following TIA or ischaemic stroke $[1,2,9,10,13]$.

The aims of this pilot, proof-of-concept, longitudinal, case-crossover study were to assess whether commencing aspirin, or changing from aspirin to clopidogrel monotherapy, significantly inhibited platelet function or activation, and to examine the relationship between platelet function and activation in response to treatment changes. We hypothesized that a novel, potentially more clinically informative 'longitudinal definition of HTPR' in response to aspirin or clopidogrel could be established with an existing test of platelet function and that platelet activation would be less marked in some patients after starting aspirin or after changing from aspirin to clopidogrel.

\section{Patients and methods}

\section{Ethics committee approval}

The study was approved by the SJH/AMNCH Local Research Ethics Committee (ref: 2008/13/02). Written informed consent or assent was obtained in all cases.

The Trinity Antiplatelet Responsiveness (TRAP) study is a prospective, longitudinal, observational study designed to detect changes in clinical and haematological parameters in patients who undergo a change in antiplatelet therapy following TIA or ischaemic stroke. Consecutive eligible patients whose treating physician opted to change their antiplatelet therapy within 4 weeks of TIA or ischaemic stroke onset from either no antiplatelet therapy to aspirin monotherapy, or from aspirin to clopidogrel monotherapy were recruited to this component of the TRAP study. Detailed clinical and neurovascular work-up was performed as described previously [6], and the underlying mechanism responsible for the TIA or ischaemic stroke was categorized according to the Trial of Org 10172 in Acute Stroke Treatment (TOAST) classification [14]. Exclusion criteria were as outlined previously, but in brief, patients were excluded if they had a history of primary intracerebral haemorrhage, myocardial infarction within the preceding 3 months, ongoing unstable angina, unstable symptomatic peripheral vascular disease, major surgery or systemic haemorrhage within the preceding 3 months (haemoglobin decrease of $>1 \mathrm{~g} / \mathrm{dl}$ in 1 day, or requiring transfusion), systemic vasculitis, underlying neoplasia, or a known bleeding or clotting diathesis, platelet count $<120 \times 10^{9} / 1$ or $>450 \times 10^{9} / 1$, urea $>10 \mathrm{mM}$ or GFR $<30 \mathrm{ml} / \mathrm{min}$, known platelet disorder, current infection (clinical signs of infection or white cell count $\left.>11 \times 10^{9} / 1\right)$ or non-steroidal antiinflammatory use other than aspirin within 14 days of recruitment [6]. Patients were recruited and followed up between September 2007 and February 2010 at our university teaching hospital that accepted primary, secondary and tertiary referrals.

Clinical and laboratory assessment was performed before (baseline) and 14 days after and at least 90 days after altering antiplatelet medication. Adherence to antithrombotic therapy was confirmed in inpatients by checking prescription charts, and by history taking alone in outpatients. Patients were phoned to encourage medication adherence in the week prior to reassessment, and reassessment was deferred for 14 days in any patients deemed possibly non-adherent to their antiplatelet regimen, or if there was any concern about re-ingestion of other nonsteroidal anti-inflammatory drugs during follow-up.

\section{Blood sampling and laboratory tests}

Blood sampling and analysis was performed using a standardized technique, as described previously $[6,15,16]$. Full blood counts (FBC) in EDTA- and $3.2 \%$ citrate-anticoagulated whole blood were performed in all subjects using a Sysmex XE-2100 haematology analyser (Sysmex UK Ltd., Milton Keynes, UK). Platelet reactivity in whole blood was assessed with the PFA-100 platelet function analyser (DadeBehring) between 2 and $2.5 \mathrm{~h}$ after venepunture [17]. The PFA-100 activates platelets by exposure to moderately high shear stress (5000-6000/s) and biochemical stimulation with collagen and either ADP (C-ADP cartridge) or epinephrine (C-EPI cartridge) $[6,16]$. The time taken for activated platelets to occlude an aperture in the cartridge is called the closure time; the maximum closure time recorded by the device is $300 \mathrm{~s}$ [6]. 
As baseline values before changing treatment were available in all patients, we established a novel, scientifically valid, 'longitudinal definition' of ex vivo HTPR on the PFA-100 using patients as their own baseline controls $[7,9,10]$. The coefficient of variation $(\mathrm{CV})$ of the C-EPI assay was $7.5 \%$ and that of the C-ADP assay was $7 \%$ in our laboratory [6]. To account for potential intra-individual variability in closure times on the device over time, HTPR in response to aspirin was defined as failure to prolong the C-EPI closure time compared with the patient's own baseline on no antiplatelet therapy by more than twice the $\mathrm{CV}$ of the $\mathrm{C}$-EPI assay, that is, failure to prolong C-EPI closure times by $>15 \%$ of the patient's baseline C-EPI closure time. Because of controversy regarding the ability of the PFA-100 to detect the inhibition of platelet function with clopidogrel [9], we investigated whether one could detect inhibition of ADP-induced platelet reactivity in response to clopidogrel with the C-ADP cartridge. HTPR in response to clopidogrel was defined as failure to prolong C-ADP closure times with clopidogrel compared with the patient's own baseline on aspirin by more than twice the CV of the C-ADP assay, that is, failure to prolong C-ADP closure times by $>14 \%$ of the patient's baseline.

Platelet activation was assessed by quantifying platelet surface CD62P and CD63 expression within $90 \mathrm{~min}$ of venepuncture $[12,15,18]$ and the percentages of circulating neutrophil-platelet, monocyte-platelet and lymphocyte-platelet complexes within $3 \mathrm{~h}$ of venepuncture [19] using previously described $[6,12,15]$ and validated methodology $[3-5,8,10,11,16,20,21]$.

\section{Data analysis}

Chi-squared tests were used to compare proportions between groups, $t$-tests for comparison of paired and unpaired parametric variables, the Wilcoxon signed rank test and the Wilcoxon rank sum test for comparison of paired and unpaired nonparametric variables, respectively, and the Kruskal-Wallis rank sum test for comparison of multiple nonparametric variables. Spearman rank-order correlation analysis assessed the relationship between HTPR and platelet activation. $P<0.05$ was considered statistically significant. All calculations were performed using $\mathrm{R}$ (http://www. r-project.org).

\section{Results}

Twenty-six patients were assessed at baseline on no medication; 25 of these patients were reassessed at 14 days and 11 at 90 days after commencing aspirin. The median interval between the index cerebrovascular event and the 90-day follow-up visit was 152 days (range 93-244 days) in these 11 patients who remained on long-term aspirin monotherapy. Ten patients had serial data at baseline, 14 days and 90 days after commencing aspirin. One patient was only assessed at baseline and at 90 days after commencing aspirin because of an unexpected wrist fracture at the 14-day follow-up phase. The remaining patients were changed to an alternative anti-thrombotic regimen (usually combination antiplatelet therapy or anticoagulation) by their treating physician prior to the 90-day assessment. In this group overall, the median daily aspirin dose was $300 \mathrm{mg}$ at 14 days and $75 \mathrm{mg}$ at 90 days.

Twenty-two patients were assessed at baseline on aspirin monotherapy and 14 days after changing to clopidogrel. Two of these patients underwent a further change in antiplatelet therapy by 90 days, so 20 patients had follow-up data at each time point. The median interval between the index cerebrovascular event and the 90-day follow-up visit was 118 days (range 95-314 days) in these 20 patients who were on long-term clopidogrel monotherapy. The median aspirin dose in this group of patients was $75 \mathrm{mg}$ daily at baseline; all patients were on $75 \mathrm{mg}$ of clopidogrel daily at 14 days and 90 days.

The clinical details of the study subjects are tabulated in Table 1, and their TIA/stroke subtypes in Table 2. No patient had a recurrent vascular event during follow-up.

Table 1 Demographic data of patients at enrolment

\begin{tabular}{lrrrrr}
\hline & $\begin{array}{l}\text { No medication } \\
\text {-aspirin } \\
(n=26)\end{array}$ & & $\begin{array}{l}\text { Aspirin- } \\
\text { clopidogrel } \\
(n=22)\end{array}$ \\
\cline { 2 - 3 } Parameter & Number & $\%$ & & Number & $\%$ \\
\hline Mean age (years) & $57( \pm 14)$ & - & $70( \pm 9)$ & - \\
Gender (M/F) & $18 / 8$ & - & $14 / 8$ & - \\
Prior stroke/TIA & 3 & 12 & 9 & 41 \\
IHD & 1 & 4 & 8 & 36 \\
Hypertension & 11 & 42 & 21 & 95 \\
Diabetes mellitus & 0 & 0 & 2 & 9 \\
A fib/flutter at enrolment & 0 & 0 & 1 & 5 \\
Family history of stroke & 13 & 50 & 7 & 32 \\
Prior DVT/PE & 1 & 4 & 0 & 0 \\
Peripheral vascular disease & 0 & 0 & 2 & 9 \\
Migraine & 5 & 19 & 4 & 18 \\
Current smoker & 7 & 27 & 5 & 23 \\
Ex-smoker & 9 & 35 & 9 & 41 \\
Never smoker & 10 & 38 & 8 & 36 \\
Statin therapy at & 5 & 19 & 17 & 77 \\
enrolment & & & & \\
\hline
\end{tabular}

TIA, transient ischaemic attack; IHD, ischaemic heart disease; A fib, atrial fibrillation; DVT, deep venous thrombosis; PE, pulmonary embolism. 
Table 2 Aetiological subtyping by TOAST classification [numbers $(\%)$ ]

\begin{tabular}{llc}
\hline $\begin{array}{l}\text { Transient ischaemic } \\
\text { attack/stroke subtype }\end{array}$ & $\begin{array}{l}\text { No meds-aspirin } \\
(n=26)\end{array}$ & $\begin{array}{l}\text { Aspirin-clopidogrel } \\
(n=22)\end{array}$ \\
\hline $\begin{array}{l}\text { Large artery } \\
\text { atherosclerotic }\end{array}$ & $0(0 \%)$ & $1(4 \%)$ \\
$\begin{array}{l}\text { Small vessel occlusion } \\
\text { Cardioembolic }\end{array}$ & $7(27 \%)$ & $3(14 \%)$ \\
Undetermined aetiology & $9(23 \%)$ & $3(14 \%)$ \\
Other determined & $4(15 \%)$ & $15(68 \%)$ \\
\hline
\end{tabular}

High on-treatment platelet reactivity after commencing aspirin

Aspirin prolonged median C-EPI closure times at 14 days $(P=0.0001 ; n=25)$ and 90 days $(P=0.002$; $n=11)$, but not median C-ADP closure times $(P \geq 0.2)$ (Table 3$)$. Using our novel longitudinal definition, $6 / 25(24 \%)$ patients at 14 days and 2/11 (18\%) at 90 days had ex vivo HTPR in response to aspirin. In the subgroup of 10 patients with serial data at each time point after commencing aspirin, there was a trend toward prolongation of the median C-EPI closure time from $87 \mathrm{~s}$ at baseline to $120 \mathrm{~s}$ at 14 days $(P=0.07)$, and a significant increase to $143 \mathrm{~s}$ at 90 days $(P=0.008)$. Six of 10 patients $(60 \%)$ retained the same 'aspirin-responder status' at both 14 days and 90 days. Platelet count did not correlate with CEPI closure time at any time point $(P \geq 0.07)$.

\section{High on-treatment platelet reactivity after changing from aspirin to clopidogrel}

Changing from aspirin to clopidogrel reduced C-EPI closure times at 14 days and 90 days compared with baseline $(P \leq 0.007)$, attributable to discontinuation of aspirin (Table 4). The median C-ADP closure time initially increased 14 days after changing to clopidogrel $(P=0.001)$, but this effect did not remain significant at 90 days $(P=0.09)$. Nine of $22(41 \%)$ patients at 14 days, and $7 / 20(35 \%)$ at 90 days demonstrated HTPR in response to clopidogrel. Platelet count did not correlate with C-EPI closure time at any time point $(P \geq 0.08)$.

\section{Effects of commencing aspirin or changing to clopidogrel on platelet activation}

Commencing aspirin did not affect platelet activation status (Table 3). Changing from aspirin to clopidogrel initially reduced circulating neutrophil-platelet complexes at 14 days $(P=0.02)$, but not at 90 days compared with baseline $(P=0.3)$, but did not influence platelet activation status otherwise (Table 4 ).

\section{Relationship between platelet function and activation}

There were no differences in CD62P or CD63 expression or percentage leucocyte-platelet complexes between patients with and without HTPR on aspirin at 14 days $(P \geq 0.1)$ or 90 days $(P \geq 0.4)$. There were no differences in CD62P or CD63 expression or percentage leucocyte-platelet complexes between patients with and without HTPR on clopidogrel at 14 days $(P \geq 0.1)$ or 90 days $(P \geq 0.1)$.

\section{Full blood count parameters following alteration of antiplatelet therapy}

There was no significant change in platelet count, platelet volume or leucocyte count in response to commencement of aspirin monotherapy (Table 5). There was a transient fall in haemoglobin concentration and haematocrit at 14 days compared with baseline $(P=0.0004)$, but these changes were not sustained at 90 days.

Changing from aspirin to clopidogrel monotherapy led to a transient reduction in leucocyte count, haemoglobin concentration, platelet count and platelet distribution width in citrated blood at 14 days compared with baseline $(P \leq 0.02)$, but these reductions were not sustained to 90 days $(P \geq 0.06)$ (Table 6$)$. The mean platelet volume in EDTA-anticoagulated blood decreased at 14 days $(P=0.02)$ and 90 days $(P=0.04)$ compared with baseline. There was no correlation between platelet count and neutrophil-platelet complexes at 14 days $(P=0.6)$.

\section{Discussion}

In agreement with prior studies [3-5,8,10,11,16,20,21], this study confirms that the PFA-100 C-EPI cartridge can detect ex vivo HTPR in patients with ischaemic CVD who are treated with aspirin. However, using our novel, scientifically valid 'longitudinal definition', the prevalence of HTPR on aspirin in this study is much lower than that anticipated from a study employing a 'cross-sectional definition' in the early phase after TIA or stroke ( $24 \%$ vs. $60 \%$, Pearson's chi-squared test for difference $P=0.003$ ) [16], and there was a trend towards a lower prevalence of HTPR on aspirin in the late phase after symptom onset also (18\% vs. $43 \%$, Fisher's exact test for difference $P=0.3)$ [16].

Our 14-day data in patients who changed from aspirin to clopidogrel are in keeping with a small, longitudinal pilot study in nine patients with ischaemic stroke which suggested a time lag of at least 12 days before one could detect initial ex vivo antiplatelet 


\begin{tabular}{|c|c|c|c|}
\hline & Baseline $(n=26)$ & 14 days $(n=25)$ & 90 days $(n=11)$ \\
\hline \multicolumn{4}{|l|}{ Platelet surface markers } \\
\hline CD62P \% & $2.02(1.35-3.07)$ & $2.54(1.54-2.68)$ & $2.57(1.11-3.36)$ \\
\hline$P$ value & & 0.4 & 1 \\
\hline CD63\% & $10.10(7.29-15.10)$ & $11.10(6.82-14.30)$ & $10.40(6.68-11.90)$ \\
\hline$P$ value & & 0.7 & 0.6 \\
\hline \multicolumn{4}{|c|}{ Leucocyte-platelet complexes } \\
\hline $\begin{array}{l}\text { Neutrophil-platelet } \\
\text { complexes } \%\end{array}$ & $2.77(2.40-3.08)$ & $2.61(2.33-2.99)$ & $2.42(2.00-2.63)$ \\
\hline$P$ value & & 0.09 & 0.2 \\
\hline $\begin{array}{l}\text { Monocyte-platelet } \\
\text { complexes } \%\end{array}$ & $4.90(3.50-5.60)$ & $4.70(3.98-5.88)$ & $4.50(3.75-5.20)$ \\
\hline$P$ value & & 0.5 & 0.6 \\
\hline $\begin{array}{l}\text { Lymphocyte-platelet } \\
\text { complexes } \%\end{array}$ & $2.40(2.08-2.66)$ & $2.49(2.25-2.66)$ & $2.10(1.94-2.34)$ \\
\hline$P$ value & & 0.5 & 0.3 \\
\hline \multicolumn{4}{|l|}{ PFA-100 } \\
\hline C-EPI (s) & $102(81-116)$ & $288(120-301)$ & $139(117-174)$ \\
\hline$P$ value & & 0.0001 & 0.002 \\
\hline C-ADP (s) & $84(73-99)$ & $91(74-103)$ & $80(71-94)$ \\
\hline$P$ value & & 0.2 & 1.0 \\
\hline
\end{tabular}

$P$ values refer to comparison between data at 14-day and 90-day follow-up versus baseline; values are medians (25-75\% interquartile range); significant $P$ values $(P<0.05)$ are highlighted in bold.

\begin{tabular}{|c|c|c|c|}
\hline & Baseline $(n=22)$ & 14 days $(n=20)$ & 90 days $(n=20)$ \\
\hline \multicolumn{4}{|l|}{ Platelet surface markers } \\
\hline $\mathrm{CD} 62 \mathrm{P} \%$ & $1.80(1.61-2.22)$ & $1.65(1.04-2.06)$ & $1.91(1.34-2.33)$ \\
\hline$P$ value & & 0.2 & 0.9 \\
\hline CD63\% & $11.30(6.73-16.58)$ & $8.48(6.56-14.30)$ & $11.90(6.75-15.85)$ \\
\hline$P$ value & & 0.3 & 0.2 \\
\hline \multicolumn{4}{|c|}{ Leucocyte-platelet complexes } \\
\hline $\begin{array}{l}\text { Neutrophil-platelet } \\
\text { complexes }\end{array}$ & $2.81(2.37-3.08)$ & $2.30(2.25-2.61)$ & $2.56(2.24-3.00)$ \\
\hline$P$ value & & 0.02 & 0.3 \\
\hline $\begin{array}{l}\text { Monocyte-platelet } \\
\text { complexes } \%\end{array}$ & $4.90(4.18-6.00)$ & $4.50(4.15-5.10)$ & $5.10(4.40-5.85)$ \\
\hline$P$ value & & 0.1 & 0.6 \\
\hline $\begin{array}{l}\text { Lymphocyte-platelet } \\
\text { complexes \% }\end{array}$ & $2.65( \pm 0.61)$ & $2.36( \pm 0.48)$ & $2.34( \pm 0.52)$ \\
\hline$P$ value & & 0.1 & 0.1 \\
\hline \multicolumn{4}{|l|}{ PFA-100 } \\
\hline C-EPI (s) & $150(127-301)$ & $114(90-129)$ & $109(96-123)$ \\
\hline$P$ value & & $<0.001$ & 0.007 \\
\hline C-ADP (s) & $82(74-96)$ & $101(86-108)$ & $98(82-112)$ \\
\hline$P$ value & & 0.001 & 0.09 \\
\hline
\end{tabular}

$P$ values refer to comparison between data at 14-day and 90-day follow-up versus baseline; values are medians $(25-75 \%$ interquartile range) or means ( \pm standard deviation); significant $P$ values $(P<0.05)$ are highlighted in bold.
Table 3 Platelet activation and platelet function in response to commencement of aspirin monotherapy in 'antiplatelet naïve' patients with cerebrovascular disease
Table 4 Platelet activation and platelet function in response to changing from aspirin to clopidogrel monotherapy following transient ischaemic attack or ischaemic stroke effects with clopidogrel, and a 4-week period before one sees its maximal antiplatelet effects [9]. Although the lack of significant findings 90 days after starting clopidogrel could relate to a type II error (only 20 of 22 patients had 90-day follow-up), our data concur with another case-crossover study [22] and indicate that the C-ADP cartridge may not reliably detect the antiplatelet effects of clopidogrel during long-term fol- low-up, even if one uses our novel longitudinal definition of HTPR on clopidogrel. Overall, a high proportion of patients with CVD (35\%) exhibited ex vivo HTPR to long-term clopidogrel on the C-ADP cartridge, but the prevalence of HTPR on clopidogrel was also much lower with our longitudinal definition than seen in studies employing cross-sectional definitions of clopidogrel HTPR in the late phase after TIA 
Table 5 Full blood count (FBC) parameters in response to commencing aspirin monotherapy following transient ischaemic attack or ischaemic stroke

\begin{tabular}{|c|c|c|c|}
\hline FBC parameter & Baseline $(n=26)$ & 14 days $(n=25)$ & 90 days $(n=11)$ \\
\hline Platelet count $\times 10^{9} / 1($ EDTA $)$ & $236( \pm 52)$ & $230( \pm 43)$ & $221( \pm 54)$ \\
\hline$P$ value & & 0.5 & 0.9 \\
\hline Platelet count $\times 10^{9} / 1$ (citrate) & $176( \pm 49)$ & $172( \pm 35)$ & $159( \pm 32)$ \\
\hline$P$ value & & 0.7 & 0.5 \\
\hline $\begin{array}{l}\text { Platelet distribution width \% } \\
\text { (EDTA) }\end{array}$ & $13.4( \pm 1.9)$ & $13.7( \pm 2.3)$ & $12.8( \pm 1.2)$ \\
\hline$P$ value & & 0.4 & 0.2 \\
\hline $\begin{array}{l}\text { Platelet distribution width \% } \\
\text { (citrate) }\end{array}$ & $11.5( \pm 1.8)$ & $11.3( \pm 1.7)$ & $11.1( \pm 1.3)$ \\
\hline$P$ value & & 0.1 & 0.5 \\
\hline $\begin{array}{l}\text { Mean platelet volume (fl) } \\
\text { (EDTA) }\end{array}$ & $10.9( \pm 0.9)$ & $11.0( \pm 1.1)$ & $10.7( \pm 0.8)$ \\
\hline$P$ value & & 0.5 & 0.7 \\
\hline $\begin{array}{l}\text { Mean platelet volume (fl) } \\
\text { (citrate) }\end{array}$ & $9.8( \pm 0.8)$ & $9.8( \pm 0.9)$ & $9.7( \pm 0.7)$ \\
\hline$P$ value & & 0.5 & 0.7 \\
\hline $\mathrm{WCC} \times 10^{9} / 1(\mathrm{EDTA})$ & $7.42( \pm 2.10)$ & $7.04( \pm 2.26)$ & $7.55( \pm 2.17)$ \\
\hline$P$ value & & 0.07 & 0.8 \\
\hline Neutrophils $\times 10^{9} / 1($ EDTA $)$ & $4.24(3.01-5.74)$ & $3.68(2.78-5.07)$ & $4.79(3.49-5.73)$ \\
\hline$P$ value & & 0.06 & 0.5 \\
\hline Monocytes $\times 10^{9} / 1($ EDTA $)$ & $0.59(0.51-0.78)$ & $0.60(0.47-0.71)$ & $0.62(0.57-0.78)$ \\
\hline$P$ value & & 1.0 & 0.2 \\
\hline Lymphocytes $\times 10^{9} / 1($ EDTA $)$ & $1.88(1.55-2.34)$ & $1.70(1.42-2.35)$ & $1.63(1.45-2.29)$ \\
\hline$P$ value & & 0.5 & 0.8 \\
\hline Haemoglobin (g/dl) (EDTA) & $15.3( \pm 1.6)$ & $14.4( \pm 1.0)$ & $15.1( \pm 0.9)$ \\
\hline$P$ value & & 0.0004 & 0.2 \\
\hline HCT (1/1) (EDTA) & $0.45( \pm 0.04)$ & $0.42( \pm 0.03)$ & $0.44( \pm 0.03)$ \\
\hline$P$ value & & 0.0004 & 0.2 \\
\hline
\end{tabular}

$P$ values refer to comparison between follow-up data at 14 days and 90 days on aspirin versus baseline values on no antiplatelet therapy; values are means $( \pm \mathrm{SD})$ or medians $(25-75 \%$ interquartile range); significant $P$ values $(P<0.05)$ are highlighted in bold.

or ischaemic stroke $[7 / 20(35 \%)$ vs. $29 / 31(93.5 \%)]$ in the study by Grau et al. $(P=0.06)[10]$. The recently licensed INNOVANCE ${ }^{\circledR}$ (Dade-Behring, Milton Keynes, UK) PFA P2Y cartridge has been designed to address the limitations of the C-ADP cartridge in detecting the antiplatelet effects of clopidogrel [22]. This cartridge was not available at the time of this study, but does deserve future inclusion in cross-sectional and longitudinal studies in CVD.

A longitudinal study design allows individual patients to act as their own controls, thus avoiding the potential influence of confounding inter-group variables, including differences in TIA and stroke subtypes or vascular risk factors that could hinder interpretation of results in case-control studies. The main variable that changes between sampling points is time from symptom onset. Thus, a longitudinal definition of HTPR has the potential to be more informative of the impact of changing antiplatelet therapy on an individual patient's platelet function in the clinical setting. We cannot comment on the clinical value of this longitudinal definition of HTPR on the PFA-100 at predicting the risk of recurrent vascular events on aspirin or clopidogrel following TIA or ischaemic stroke because none of the patients had recurrent vascular events during follow-up in this study.

In agreement with previous pilot studies, CD62P and CD63 expression and leucocyte-platelet complex formation did not change after commencing aspirin [10], and CD62P and CD63 expression did not change after switching from aspirin to clopidogrel [23]. Although there was a transient reduction in neutrophil-platelet complex formation 14 days after changing to clopidogrel, this was not sustained at 90 days. Although these findings could represent a type I error at 14 days, or a type II error at 90 days, the results of this and other pilot studies indicate that the measurement of changes in platelet activation status with unstimulated whole-blood flow cytometry, as a measure of 'laboratory responsiveness' to antiplatelet therapy, is unlikely to be informative at predicting HTPR in ischaemic CVD. Preliminary data from control subjects on clopidogrel and patients with ischaemic heart disease on aspirin and clopidogrel indicate that flow cytometry protocols that assess 'inducible platelet activation/reactivity' have the 


\begin{tabular}{|c|c|c|c|}
\hline FBC parameter & $\begin{array}{l}\text { Baseline } \\
(n=22)\end{array}$ & $\begin{array}{l}14 \text { days } \\
(n=20)\end{array}$ & $\begin{array}{l}90 \text { days } \\
(n=20)\end{array}$ \\
\hline Platelet count $\times 10^{9} / 1(\mathrm{EDTA})$ & $215( \pm 45)$ & $200( \pm 38)$ & $211( \pm 41)$ \\
\hline$P$ value & & 0.01 & 0.5 \\
\hline Platelet count $\times 10^{9} / 1$ (citrate) & $158( \pm 39)$ & $151( \pm 28)$ & $161( \pm 32)$ \\
\hline$P$ value & & 0.2 & 0.8 \\
\hline Platelet distribution width \% (EDTA) & $13.2( \pm 1.9)$ & $12.7( \pm 1.9)$ & $12.9( \pm 1.9)$ \\
\hline$P$ value & & 0.02 & 0.08 \\
\hline Platelet distribution width \% (citrate) & $11.1(9.9-12.3)$ & $11.150(9.7-11.9)$ & $11.3(10.2-12.6)$ \\
\hline$P$ value & & 0.3 & 0.6 \\
\hline Mean platelet volume (fl) (EDTA) & $11.0(10.0-11.4)$ & $10.8(9.8-11.3)$ & $10.7(10.1-11.2)$ \\
\hline$P$ value & & 0.02 & 0.04 \\
\hline Mean platelet volume (fl) (citrate) & $9.8(9.2-10.1)$ & $9.9(9.0-10.3)$ & $10.1(9.3-10.5)$ \\
\hline$P$ value & & 0.9 & 0.7 \\
\hline $\mathrm{WCC} \times 10^{9} / 1(\mathrm{EDTA})$ & $6.84( \pm 1.84)$ & $6.17( \pm 1.54)$ & $6.54( \pm 1.50)$ \\
\hline$P$ value & & 0.02 & 0.09 \\
\hline Neutrophils (EDTA) & $3.8(3.0-5.2)$ & $3.7(2.9-4.5)$ & $3.6(3.1-4.4)$ \\
\hline$P$ value & & 0.3 & 1.0 \\
\hline Monocytes $\times 10^{9} / 1($ EDTA $)$ & $0.66( \pm 0.21)$ & $0.55( \pm 0.15)$ & $0.59( \pm 0.16)$ \\
\hline$P$ value & & 0.009 & 0.08 \\
\hline Lymphocytes $\times 10^{9} / 1($ EDTA $)$ & $1.92( \pm 0.72)$ & $1.70( \pm 0.65)$ & $1.82( \pm 0.67)$ \\
\hline$P$ value & & 0.003 & 0.06 \\
\hline Haemoglobin (g/dl) (EDTA) & $14.1( \pm 1.6)$ & $13.8( \pm 1.6)$ & $14.0( \pm 1.5)$ \\
\hline$P$ value & & 0.01 & 0.09 \\
\hline HCT (1/1) (EDTA) & $3.8(3.0-5.2)$ & $3.7(2.9-4.5)$ & $3.6(3.1-4.4)$ \\
\hline$P$ value & & 0.3 & 1.0 \\
\hline
\end{tabular}

Table 6 Full blood count (FBC) parameters in response to changing from aspirin to clopidogrel monotherapy following transient ischaemic attack or ischaemic stroke

$P$ values refer to comparison between follow-up data at 14 days and 90 days on clopidogrel versus baseline values on aspirin; values are means $( \pm \mathrm{SD})$ or medians $(25-75 \%$ interquartile range); significant $P$ values $(P<0.05)$ are highlighted in bold.

potential to be clinically informative [24]. Flow cytometry techniques also have the ability to select subsets of platelets that are more procoagulant than others [25] and may help guide future therapy in ischaemic CVD. We fully acknowledge that our pilot study only included a small number of patients on aspirin monotherapy who had follow-up data at the 90-day phase. Therefore, we may not have identified significant changes in platelet activation because of a type II error. Allowing for this, a small but statistically significant change in unstimulated $\mathrm{CD} 62 \mathrm{P}$ or CD63 surface expression is unlikely to be clinically useful in monitoring patients with CVD undergoing antiplatelet treatment changes.

Changing from aspirin to clopidogrel monotherapy resulted in a reduction in all $\mathrm{FBC}$ parameters at 14 days, and a reduction in mean platelet volume at both 14 and 90 days. These novel data in patients with ischaemic CVD indicate that clopidogrel may exert a transient, 'global myelosuppressive effect' following TIA or ischaemic stroke. The persistent reduction in platelet size with clopidogrel, which to our knowledge has not been noted before, may have a beneficial influence on platelet reactivity in the laboratory and clinical setting during follow-up, because smaller platelets are believed to be less reactive than larger platelets [26].
This study answers some important questions regarding ex vivo HTPR on aspirin or clopidogrel in patients following TIA and ischaemic stroke, but has some limitations. The small number of patients with long-term follow-up data at 90 days after changing from no medication to aspirin limits our ability to draw definitive conclusions from these data because of the possibility of a type I error. The recently licensed INNOVANCE ${ }^{\circledR}$ PFA P2Y cartridge, which has been designed to address the limitations of the C-ADP cartridge in detecting the antiplatelet effects of clopidogrel [22], was not available at the time this study was conducted, so we cannot comment on its potential value in monitoring patients with ischaemic CVD on clopidogrel at present. Furthermore, the clinical significance of HTPR on the PFA-100 or any other platelet function device in ischaemic CVD is unclear at present, as discussed earlier.

In conclusion, the prevalence of ex vivo HTPR in patients with ischaemic CVD is much lower if one employs our 'novel longitudinal definition' than a 'traditional cross-sectional definition' of HTPR. On the basis of the interesting data from this pilot, proofof-concept study, a large, adequately powered, longitudinal, multicentre study, using a panel of existing and novel platelet function tests [20,22,24,27-31], has been planned to assess the value of both longitudinal 
and cross-sectional definitions of HTPR, at different shear rates, at predicting the risk of recurrent vascular events in ischaemic CVD. These studies are required to determine whether ischaemic CVD patients with HTPR are at higher risk of recurrent clinical or subclinical vascular events than patients without HTPR ex vivo. Furthermore, one needs to investigate whether patients with very marked inhibition of platelet function have a higher risk of bleeding complications than patients with HTPR. Such data have the potential to facilitate optimized secondary prevention with costeffective, individualized antiplatelet regimens following TIA or stroke.

\section{Acknowledgements}

Study design, data collection, analysis, interpretation or manuscript writing were carried out by WO Tobin, JA Kinsella and DJH McCabe. Data collection, interpretation and critical revision of the manuscript for important intellectual content were performed by $\mathrm{T}$ Coughlan, DR Collins, D O'Neill, B Egan, S Tierney, TM Feeley and RP Murphy. Dr Tobin was funded by an IICN-Serono Fellowship, Meath Foundation, Lundbeck Neurosciences Bursary programme, Merck Serono Ireland, Brennan and Company Ireland and Biogen Idec Ireland Limited. Dr Kinsella was funded by the Stanley Thomas Johnson Foundation, Bayer Schering Ireland, Pfizer Ireland and Elitech UK. Dr McCabe's research was supported by a grant from the Programme for Research in Third Level Institutions in Ireland (Cycle 4), co-funded by the European Regional Development Fund.

\section{Conflict of interests}

The authors declare no financial or other conflict of interests.

\section{References}

1. Serebruany VL, Malinin AI, Sane DC, et al. Magnitude and time course of platelet inhibition with aggrenox and aspirin in patients after ischemic stroke: the AGgrenox versus aspirin therapy evaluation (AGATE) trial. Eur $J$ Pharmacol 2004; 499: 315-324.

2. Serebruany VL, Malinin AI, Pokov AN, Hanley DF. Antiplatelet profiles of the fixed-dose combination of extended-release dipyridamole and low-dose aspirin compared with clopidogrel with or without aspirin in patients with type 2 diabetes and a history of transient ischemic attack: a randomized, single-blind, 30-day trial. Clin Ther 2008; 30: 249-259.

3. Alberts MJ, Bergman DL, Molner E, Jovanovic BD, Ushiwata I, Teruya J. Antiplatelet effect of aspirin in patients with cerebrovascular disease. Stroke 2004; 35: $175-178$.
4. Grundmann K, Jaschonek K, Kleine B, Dichgans J, Topka H. Aspirin non-responder status in patients with recurrent cerebral ischemic attacks. J Neurol 2003; 250: 63-66.

5. Harrison P, Segal H, Blasbery K, Furtado C, Silver L, Rothwell PM. Screening for aspirin responsiveness after transient ischemic attack and stroke: comparison of 2 point-of-care platelet function tests with optical aggregometry. Stroke 2005; 36: 1001-1005.

6. Tobin WO, Kinsella JA, Collins DR, et al. Enhanced ex vivo inhibition of platelet function following addition of dipyridamole to aspirin after transient ischaemic attack or ischaemic stroke: first results from the TRinity AntiPlatelet Responsiveness (TRAP) Study. Br J Haematol 2011; 152: 640-647.

7. Kotzailias N, Kirsten E, Thomas S, et al. Clopidogrelinduced platelet inhibition cannot be detected by the platelet function analyzer-100 system in stroke patients. J Stroke Cerebrovasc Dis 2007; 16: 199-202.

8. Zytkiewicz M, Gielwanowska L, Wojtasinska E, Psuja $\mathrm{P}$, Zawilska K. Resistance to acetylsalicylic acid in patients after ischemic stroke. Pol Arch Med Wewn 2008; 118: 727-733.

9. Raman S, Jilma B. Time lag in platelet function inhibition by clopidogrel in stroke patients as measured by PFA-100. J Thromb Haemost 2004; 2: 2278-2279.

10. Grau AJ, Reiners S, Lichy C, Buggle F, Ruf A. Platelet function under aspirin, clopidogrel, and both after ischemic stroke. Stroke 2003; 34: 849-854.

11. von Lewinski F, Riggert J, Paulus W. Towards a rationale of platelet aggregation monitoring in stroke prophylaxis? J Stroke Cerebrovasc Dis 2009; 18: 111-115.

12. McCabe DJH, Harrison P, Mackie IJ, et al. Increased platelet count and leucocyte-platelet complex formation in acute symptomatic compared with asymptomatic severe carotid stenosis. J Neurol Neurosurg Psychiatry 2005; 76: 1249-1254.

13. Serebruany VL, Malinin AI, Ziai W, et al. Effects of clopidogrel and aspirin in combination versus aspirin alone on platelet activation and major receptor expression in patients after recent ischemic stroke: for the plavix use for treatment of stroke (PLUTO-Stroke) trial. Stroke 2005; 36: 2289-2292.

14. Adams HP Jr, Bendixen BH, Kappelle LJ, et al. Classification of Subtype Of Acute Ischemic Stroke. Definitions for use in a multicenter clinical trial. TOAST. Trial of Org 10172 in acute stroke treatment. Stroke 1993; 24: 35-41.

15. McCabe DJ, Harrison P, Mackie IJ, et al. Platelet degranulation and monocyte-platelet complex formation are increased in the acute and convalescent phases after ischaemic stroke or transient ischaemic attack. $\mathrm{Br} J$ Haematol 2004; 125: 777-787.

16. McCabe DJ, Harrison P, Mackie IJ, et al. Assessment of the antiplatelet effects of low to medium dose aspirin in the early and late phases after ischaemic stroke and TIA. Platelets 2005; 16: 269-280.

17. Kundu SK, Heilmann EJ, Sio R, Garcia C, Davidson $\mathrm{RM}$, Ostgaard RA. Description of an in vitro platelet function analyzer - PFA-100. Semin Thromb Hemost 1995; 21(Suppl 2): 106-112.

18. Joseph JE, Harrison P, Mackie IJ, Isenberg DA, Machin SJ. Increased circulating platelet-leucocyte complexes and platelet activation in patients with antiphospholipid 
syndrome, systemic lupus erythematosus and rheumatoid arthritis. Br J Haematol 2001; 115: 451-459.

19. Shattil SJ, Cunningham M, Hoxie JA. Detection of activated platelets in whole blood using activation-dependent monoclonal antibodies and flow cytometry. Blood 1987; 70: $307-315$.

20. Harrison P, Segal H, Silver L, Syed A, Cuthbertson FC, Rothwell PM. Lack of reproducibility of assessment of aspirin responsiveness by optical aggregometry and two platelet function tests. Platelets 2008; 19: 119-124.

21. Boncoraglio GB, Bodini A, Brambilla C, Corsini E, Carriero MR, Parati EA. Aspirin resistance determined with PFA-100 does not predict new thrombotic events in patients with stable ischemic cerebrovascular disease. Clin Neurol Neurosurg 2009; 111: 270-273.

22. Linnemann B, Schwonberg J, Rechner AR, Mani H, Lindhoff-Last E. Assessment of clopidogrel nonresponse by the PFA-100 system using the new test cartridge INNOVANCE PFA P2Y. Ann Hematol 2010; 89: 597-605.

23. Frelinger AL, III, Barnard MR, Fox ML, Michelson AD. The platelet activity after clopidogrel termination (PACT) study. Circ Cardiovasc Interv 2010; 3: 442-449.
24. Fox SC, May JA, Shah A, Neubert U, Heptinstall S. Measurement of platelet P-selectin for remote testing of platelet function during treatment with clopidogrel and/ or aspirin. Platelets 2009; 20: 250-259.

25. Prodan CI, Stoner JA, Cowan LD, Dale GL. Lower coated-platelet levels are associated with early hemorrhagic transformation in patients with non-lacunar brain infarction. J Thromb Haemost 2010; 8: 1185-1190.

26. Harrison P, Robinson MS, Mackie IJ, Machin SJ. Reticulated platelets. Platelets 1997; 8: 379-383.

27. Sun B, Tandon NN, Yamamoto N, Yoshitake M, Kambayashi J. Luminometric assay of platelet activation in 96-well microplate. Biotechniques 2001; 31: 1174, $1176,1178$.

28. Moran N, Kiernan A, Dunne E, Edwards RJ, Shields DC, Kenny D. Monitoring modulators of platelet aggregation in a microtiter plate assay. Anal Biochem 2006; 357: 77-84.

29. VerifyNow Aspirin. Instructions for use [Package Insert]. Accumetrics Inc., 2010.

30. VerifyNow P2Y12. Instructions for use [Package Insert]. Accumetrics Inc., 2010.

31. VerifyNow IIb/IIIa. Instructions for use [Package Insert]. Accumetrics Inc., 2010. 